\title{
MR Diffusion Histology and Micro-Tractography Reveal Mesoscale Features of the Human Cerebellum
}

\author{
Flavio Dell'Acqua • Istvan Bodi • David Slater • \\ Marco Catani • Michel Modo
}

Published online: 2 August 2013

(C) The Author(s) 2013. This article is published with open access at Springerlink.com

\begin{abstract}
After 140 years from the discovery of Golgi's black reaction, the study of connectivity of the cerebellum remains a fascinating yet challenging task. Current histological techniques provide powerful methods for unravelling local axonal architecture, but the relatively low volume of data that can be acquired in a reasonable amount of time limits their application to small samples. State-of-the-art
\end{abstract}

F. Dell'Acqua $(\varangle) \cdot$ D. Slater $\cdot$ M. Catani

Natbrainlab, Department of Neuroimaging, King's College

London, London SE5 8AF, UK

e-mail: flavio.dellacqua@kcl.ac.uk

F. Dell'Acqua

NIHR Biomedical Research Centre for Mental Health at South London and Maudsley NHS Foundation Trust and Institute of Psychiatry, King's College London, London, UK

I. Bodi

King's College Hospital NHS Foundation Trust,

Department of Clinical Neuropathology, King's College London,

London SE5 9RS, UK

\section{Catani}

Natbrainlab, Department of Forensic and Neurodevelopmental

Sciences, King's College London, London SE5 8AF, UK

M. Modo

Department of Neuroscience, King's College London, London SE5 8AF, UK

\section{Modo}

Department of Radiology, University of Pittsburgh, Pittsburgh, PA 15203, USA

M. Modo $(\bowtie)$

McGowan Institute for Regenerative Medicine, University of Pittsburgh, Pittsburgh, PA 15203, USA

e-mail: modomm@upmc.edu

M. Modo

Department of Bioengineering, University of Pittsburgh, Pittsburgh, PA 15203, USA in vivo magnetic resonance imaging (MRI) methods, such as diffusion tractography techniques, can reveal trajectories of the major white matter pathways, but their correspondence with underlying anatomy is yet to be established. Hence, a significant gap exists between these two approaches as neither of them can adequately describe the three-dimensional complexity of fibre architecture at the level of the mesoscale (from a few millimetres to micrometres). In this study, we report the application of MR diffusion histology and micro-tractography methods to reveal the combined cytoarchitectural organisation and connectivity of the human cerebellum at a resolution of $100-\mu \mathrm{m}$ ( $2 \mathrm{nl} /$ voxel volume). Results show that the diffusion characteristics for each layer of the cerebellar cortex correctly reflect the known cellular composition and its architectural pattern. Micro-tractography also reveals details of the axonal connectivity of individual cerebellar folia and the intracortical organisation of the different cerebellar layers. The direct correspondence between MR diffusion histology and micro-tractography with immunohistochemistry indicates that these approaches have the potential to complement traditional histology techniques by providing a non-destructive, quantitative and three-dimensional description of the microstructural organisation of the healthy and pathological tissue.

Keywords Diffusion tensor imaging · Tractography · Cerebellum $\cdot$ Post-mortem $\cdot$ Human $\cdot$ MR diffusion histology $\cdot$ Connectome $\cdot$ Multi-scale $\cdot$ Micro-tractography

\section{Introduction}

The year 2013 marks the 140th anniversary of Camillo Golgi's discovery of the black reaction or "reazione nera" [1]. By applying his technique to the cerebellum, Golgi was able to describe the morphology of individual cells and, for the first time, the complexity of their dendritic arborisation and axonal "reticular" architecture. This work paved the way for Santiago 
Ramon y Cajal's refined studies of histology and cerebellar connectivity and the definitive validation of the neuron doctrine $[2,3]$. As a result of these pioneering anatomical works and subsequent physiological studies $[4,5]$, neuroscientists were able to integrate theoretical work with clinical observations. This led to the development of first computational models of cerebellar networks $[6,7]$ that were fundamental for understanding the basis for cerebellar physiology and motor control [8] and marked the beginning of modern Computational Neuroscience [9]. Studies of cerebellar connectivity were further extended to extra-cerebellar circuits with the use of viral tracers $[10,11]$ in animal models, and the demonstration of parallel cerebellar-cortical loops consolidated the link between the cerebellum and frontal lobes. This experimental evidence supported the clinical observation of disorders of cognition and behaviour associated with cerebellar pathology [12, 13]. More recent in vivo neuroimaging studies have used high-resolution structural MR imaging and high-field MR scanners to depict very fine details of the human cerebellar anatomy [14, 15], whereas diffusion-based tractography has allowed the study of major cerebellar pathways [16] in autism, schizophrenia and other disorders [17-21].

However, a fundamental gap between histological and current in vivo neuroimaging studies remains. While a detailed cytoarchitectural description of human cerebellar histology has long been established, a seamless integration with larger scale networks is incomplete. The study of connectivity poses a multi-scale problem ranging from major white matter tracts at the macroscale (centimetres to millimetres) to individual axons or even single synaptic connections at the micro/nanoscale (micrometres to nanometres). In between these two extremes is the mesoscale (millimetres to micrometres) comprised of cell assemblies and small groups of axons [22-24]. Each level of organisation requires appropriate tools to decipher connections, and current methods are not suitable to visualise connections at all levels.

Traditional fibre-tracing studies provide a reliable identification of neuronal connections at the microscale (microscopy) and in some cases at the nanoscale (electron microscopy), but are limited to these scales due to the thin sectioning in two dimensions [25]. Therefore, tracing over long distances is a major challenge, and three-dimensional reconstructions are problematic due to tissue distortions, small sample sizes and cutting artefacts [26, 27]. MRI-based tracers, such as manganese, can overcome some of these limitations [28] and provide an additional macroscale assessment of fibre tracts, but are limited in providing a system view and cannot be applied to humans due to its neurotoxicity [29]. Although histological tracing studies in primates have been instrumental in establishing basic principles and evolutionary aspects of brain development, it remains difficult to draw reliable inferences about fibre tracts in humans based purely on those from other species [30-33]. This is particularly relevant in light of evidence suggesting a role of the cerebellum in higher cognition and behaviour that is unique to humans (e.g. language and executive functions) [11, 34-36].

Non-invasive white matter tracking in humans is routinely performed using diffusion-based MRI [37]. Here, the anisotropic movement of water molecules along fibre tracts allows a tractographical reconstruction of major white matter trajectories and offers the possibility of mapping large-scale networks in the living human brain [38-42]. However, two major limitations affect in vivo diffusion imaging today: the low spatial resolution of diffusion data achievable with current clinical hardware and the lack of histological validation for tractography and diffusion indices. Low spatial resolution limits current investigations to main brain features of white matter pathways and sometimes this results in partial or incorrect descriptions of the underlying tissue microstructure due to the amalgamation of properties from different tissues within the same voxel [43]. Also, little is known about the true correspondence between tractography reconstructions, diffusion indices and the underlying tissue organisation that can be determined, for example, from histology studies in healthy and pathological tissue [44]. In vivo methods are therefore currently unable to provide an adequate description of the small and local cortical networks between close regions leaving the mesoscale a largely unexplored domain.

A way to overcome these limitations is to perform diffusion imaging on the post-mortem human brain using preclinical scanners. By acquiring post-mortem data, imaging results can be promptly validated with histological analyses, and it is possible to investigate the direct correspondence between the diffusion signal and the real underlying microstructure [44-46]. Post-mortem imaging also removes some of the intrinsic time constraints of in vivo imaging, allowing significantly longer scan times that, combined with the more powerful hardware of preclinical scanners, give access to much higher spatial resolutions and signal to noise ratios [47-49]. Previous studies using ex vivo human tissue have shown that "grey matter" characteristics, such as cell layers, can be revealed using diffusion-based MRI [49]. In this study, we extend these results and show that diffusion MRI can be further applied to describe the combined cytoarchitectural organisation and connectivity characteristics at the mesoscale level. By combining complementary information about the microstructural organisation of the tissue, including fractional anisotropy (FA), apparent diffusion coefficient (ADC) maps and diffusion-weighted images (DWI) $/ T_{2}$-weighted images, we have created novel MR diffusion histology maps. When applied to a fixed post-mortem human cerebellum, these were able to show different mesoscale laminar features and their structural properties. To validate these results, we verified our findings with immunohistochemistry images of the corresponding region and with the known cytoarchitectural organisation of 
the cerebellum. Additionally, using tractography reconstructions based on diffusion tensor imaging (DTI) data, we have reconstructed, for the first time, the main trajectories of axonal projections within individual cerebellar folia and exposed the fine details of intra-cortical structural organisation within the cerebellar cortex. Advantages and limitations of these approaches are finally presented in the discussion.

\section{Methods}

\section{Post-mortem Tissue}

The cerebellum of an 11-year-old female was made available from the Clinical Neuropathology Department at King's College Hospital under the hospital post-mortem consent for medical and genetic research. The brain was extracted $45 \mathrm{~h}$ after death from acute haemorrhage due to a pilocytic astrocytoma of the spinal cord (grade I) and fixed by immersion in $10 \%$ neutral buffered formalin for 4 weeks. After fixation, the cerebellum was separated from the brainstem by severing the cerebellar peduncles. MR images were initially acquired from the entire cerebellum and then from smaller samples. The time from death to scan, or scan interval [48], was 3 months. To reduce susceptibility artefacts and dehydration of the sample over time, samples were acquired immersed in a proton-free fluid, Fomblin (Solvay Solexis Inc.). To avoid air bubbles, immersion in Fomblin was performed $24 \mathrm{~h}$ before scanning. Following Miller et al. [48], tissue samples were not presoaked in a phosphate buffer solution before scanning to minimise long-term tissue changes. After imaging, the samples were returned to the Clinical Neuropathology Department for immunohistochemistry analysis.

\section{MRI Acquisition}

MRI data were acquired using a $7 \mathrm{~T}$ Varian/Agilent (Palo Alto, USA) system using a 105/60 HD gradient system and a 39-mm quadrature volume RF coil. A Fast Spin Echo (FSE) $T_{2}$-weighted sequence was acquired to provide a high-resolution structural reference images for planning subsequent diffusion imaging scans $(\mathrm{TR}=4,000 \mathrm{~ms}, \mathrm{TE}=10 \mathrm{~ms}$, averages $=16, \quad F O V=30 \times 30 \mathrm{~mm}$, matrix $=128 \times 128,45$ slices at $0.6 \mathrm{~mm}$ thickness and no gap). Diffusion MRI data were acquired using a Pulsed Gradient Spin Echo sequence $(\mathrm{TR}=3,600 \mathrm{~ms}, \mathrm{TE}=36 \mathrm{~ms}, G=500 \mathrm{mT} / \mathrm{m}, \delta=4 \mathrm{~ms}$, $\Delta=15 \mathrm{~ms}, b$ value $\approx 4,200 \mathrm{~s} / \mathrm{mm}^{2}$, averages $=170,3$ nondiffusion-weighted volumes (b0) and 30 diffusion-weighted volumes/directions, $\mathrm{FOV}=51.2 \times 6.4 \mathrm{~mm}$, matrix $512 \times 32$, 65 slices at $0.1 \mathrm{~mm}$ thickness and no gap). Volume planning was set up to obtain the highest spatial resolution $(100 \mu \mathrm{m}$ in plane) orthogonal to most of the cerebellar folia as visible from the FSE images. Saturation bands were placed at the limit of the sample FOV along the phase direction. $\mathrm{SNR}_{\mathrm{b} 0}$ in the tissue was above 12 . Total scan time was approximately $140 \mathrm{~h}$.

\section{MRI Data Processing}

Diffusion data were processed using ExploreDTI (www. exploredti.com). Data were first pre-processed correcting for eddy current distortions and for long-term movements of the sample. Diffusion tensors were estimated using the full b-matrix, including the effect of the imaging gradient and applying a non-linear least square approach [50]. Mean ADC, FA, non-diffusion/ $\mathrm{T}_{2}$-weighted (T2w) image and average DWI maps were generated. Tractography was performed using an Euler-like streamline algorithm with a step-size of $50 \mu \mathrm{m}$ and the entire imaged sample as seed region. FA thresholds were set to 0.07 to initiate and continue tracking. The lower FA threshold relative to the usual in vivo threshold (e.g. FA $=0.15 / 0.20$ ) was required due to the intrinsically low diffusivity and anisotropy of the formalin-fixed sample. Angular threshold was set equal to $45^{\circ}$.

\section{Immunohistochemistry}

After cutting histological sections $(50 \mu \mathrm{m})$ on a freezing microtome, sections were incubated overnight $\left(21^{\circ} \mathrm{C}\right)$ to detect white matter based on rat anti-myelin basic protein (MBP, 1:100, Abcam), neurons based on rabbit anti-neurofilament $160 \mathrm{kD}(1: 1,000$, Abcam), Purkinje cells using a rabbit anticalbindin (1:200, Abcam), and Bergmann glia using mouse anti-glial fibrillary acid protein (GFAP, 1:3,000, Sigma) primary antibodies. Appropriate secondary antibodies (Alexa350, 488 and 546, Molecular Probes) were applied for $1 \mathrm{~h}$ prior to three washes and coverslipping with Vectashield for fluorescence (Vector Labs). Where appropriate, Hoechst was used as a blue nuclear stain. Sections were visualised on a Zeiss Axioplan.

\section{Results}

Cytoarchitectural Basis of Diffusion Anisotropy

In the cerebellum, key neuroanatomical features of each folium, which represent the smallest structure on the surface of the cerebellum, consist of a white matter medullary layer (WM), an inner granule cell layer (cortical layer III), a middle Purkinje cell layer (II) and an outer molecular cell layer (I). These features vary in size from $25-40 \mu \mathrm{m}$ for the Purkinje cell layer to $1 \mathrm{~mm}$ for the larger strand of white matter within a folium. With a spatial resolution of $100 \times 100 \times 200 \mu \mathrm{m}$, the data acquired in our experiment allow the visualisation of mesoscale histological features within individual cerebellar folia (Fig. 1). At this resolution, DWI, ADC and FA maps reveal different cytoarchitectural aspects of cerebellar 
organisation and offer the opportunity to perform quantitative analysis of individual layers. For example, as the axonal white matter (WM) projections from the cerebellar folia disperse within the granule cell layer, the ADC increases while the FA decreases, suggesting an increase of water mobility due to changes in the structural organisation of fibres (e.g. loss of parallel pattern, branching, and reduced myelination). In the molecular layer (I), a uniform increase in FA and decrease in $\mathrm{ADC}$ is observed, suggesting a higher cellular density and possibly a more ordered axonal packing within this layer. By pseudo-colouring each MR image and merging these into a single image akin to fluorescence histology, an MR diffusion histology image was created to highlight anatomical features, such as the laminar organisation of the cerebellar cortex and tissue compartmentalisation between white and grey matter. These results appear consistent and uniform across the entire imaged sample. Direct comparison of MR diffusion histology images with immunohistochemical images of the same region corroborates these findings and confirms that both imaging techniques visualise similar cytoarchitectural features of the cerebellar folia, although at a different spatial resolution (Fig. 1).

The cellular layer specificity of these MR images is further shown by a quantitative analysis of changes in signal intensity
(Fig. 2). The ADC of the granule cell layer (III) uniformly increases starting from low ADC values close to white matter and reaches a maximum in proximity or at the level of layer II. The high diffusivity (i.e. high water molecular mobility) in this region may suggest a decrease of tissue density or an increase of extracellular space. It is worth observing that this increase in ADC partially resembles the decrease of MBP contrast observed in the granular layer as show in Fig. 1. ADC sharply decreases within the molecular layer suggesting a sudden change in microstructural organisation that reduces the average mobility of water in this layer. The intermediate Purkinje cell layer (II) is consistently visible on $\mathrm{T}_{2}$-weighted images as a brighter separation between the larger layer III and layer I. In the MR diffusion histology image, however, this layer is not always visible due to partial volume effects and ADC values similar to layer III.

Tractography of Cerebellar Pathways from Macro- to Mesoscale

The ability to provide a three-dimensional description of the microstructural organisation of biological tissues is a unique feature of diffusion imaging. However, conventional in vivo

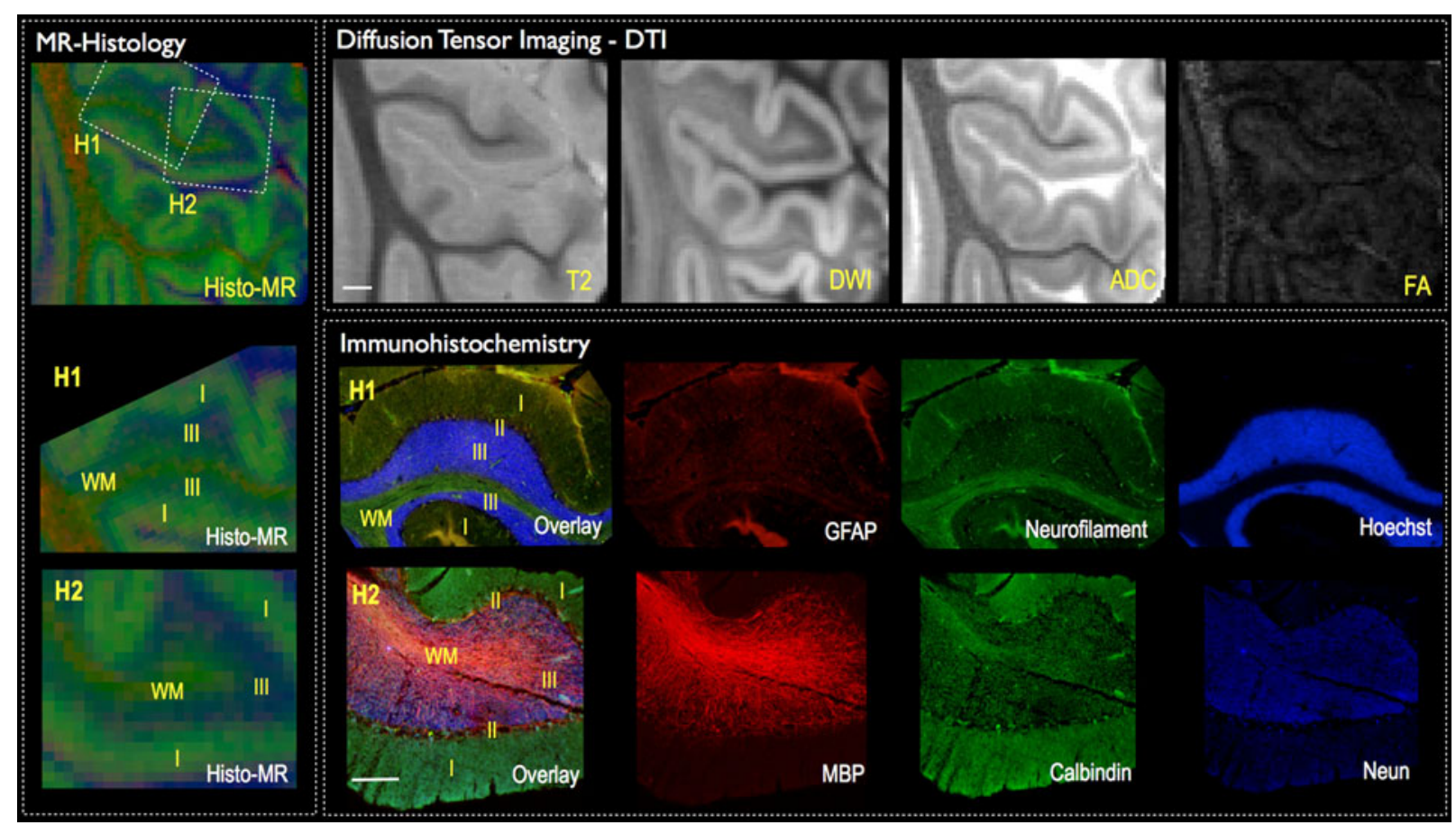

Fig. 1 MR diffusion histology and immunohistochemistry. The cytoarchitectonic features of the cerebellar cortex are shown by diffusion imaging maps at $100 \mu \mathrm{m}$ resolution. White matter $(W M)$, granular layer (III), Purkinje cell layer (II) and molecular layer $(I)$ can be discriminated across the different $\mathrm{T}_{2}$-weighted images, diffusion-weighted images $(D W I)$, apparent diffusion coefficient $(A D C)$ and fractional anisotropy (FA) contrasts (scale bar $=1 \mathrm{~mm}$ ). A pseudo-colour visualisation of these maps is obtained by combining together FA (red), DWI (green) and ADC (blue) maps to mimic immunohistochemistry results and obtain a similar
MR diffusion histology map. The comparison between MR diffusion histology and immunohistochemistry of the same region shows a good matching of features. Specifically, white matter tracts correspond to regions rich in myelin basic protein (MBP) and neurofilament, whereas a high cellular density, as indicated by the nuclear dye Hoechst, characterised the granular cell layer $(I I I)($ scale bar $=250 \mu \mathrm{m})$. These cells were predominantly neurons (NeuN+cells), whereas the Purkinje cell layer (II) was defined by calbindin+ cells. However, the homogenous distribution of astrocytes (GFAP + cells) was not associated with MRI-detectable features 

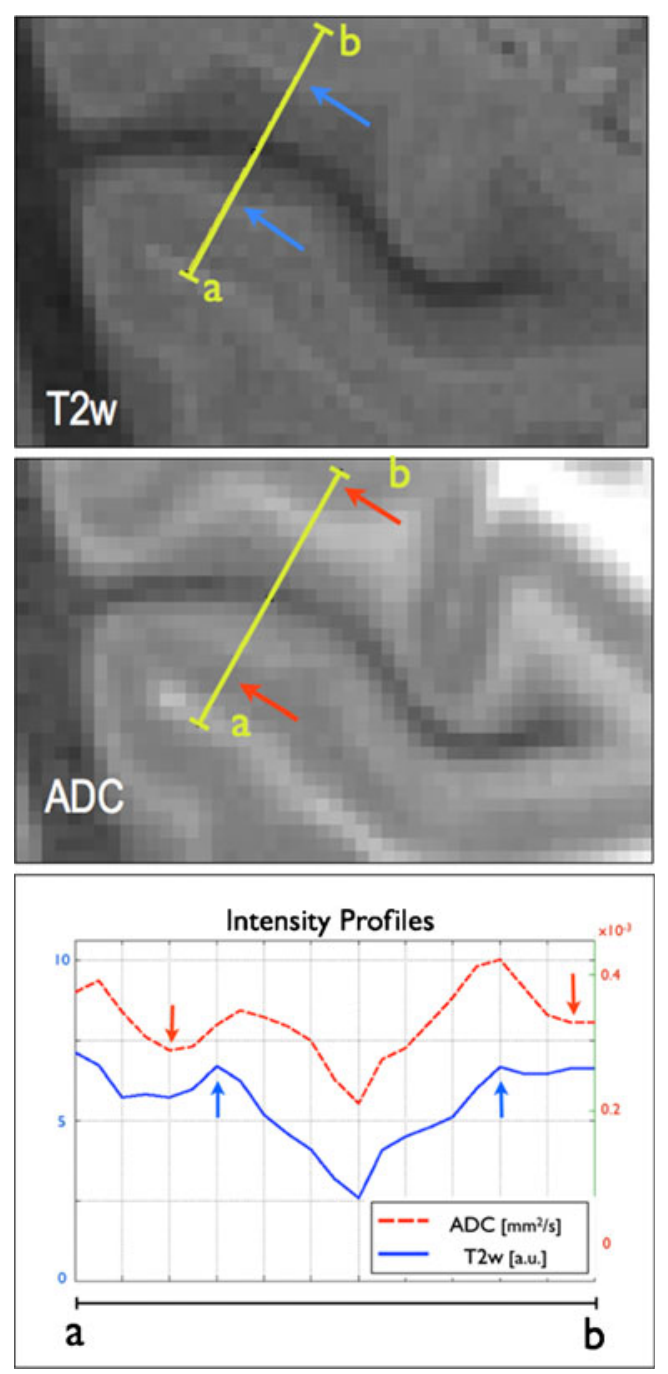

Fig. 2 Laminar specificity of MR diffusion histology images. An intensity plot reveals laminar features on a $\mathrm{T}_{2}$-weighted (T2w) image (blue line) and $\mathrm{ADC}$ (red line) map. On the $\mathrm{T} 2 \mathrm{w}$ image, a hyperintense line separates the molecular cell layer (I) and granular cell layer (III). This cytoarchitectural feature (blue arrows) represents the Purkinje cell layer (II). However, this layer is not evident on the ADC map, but here the molecular (I), granular cell layer (III, red arrows) and white matter can be clearly separated as distinct regions. These signal intensity changes further corroborate the ability of diffusion MRI to discriminate cytoarchitectural features at high resolution and characterise microstructural properties of each individual cerebellar layer

or post-mortem tractography performed with a relatively large voxel size can only describe macroscale features and does not provide a level of detail that can be integrated with microscale histology because multiple histological features are mixed within a single voxel (Fig. 3a). In contrast, microtractography performed at $100 \mu \mathrm{m}$ allows the visualisation of mesoscale features that can be directly integrated with the histological organisation of the cerebellum. At this resolution, it is possible to visualise white matter tracts running into individual cerebellar folia and dispersing inside the granule cell layer. It is also possible to reconstruct cerebellar parallel fibres as a structural continuum of parallel orientations running inside the molecular layer (Fig. 3b). In this figure, we show all streamlines passing through either a region of interest (ROI) delineating the molecular layer or through a ROI defining the white matter medullary layer. Both ROIs were selected on two consecutive image slices at the centre of the sample and no exclusion ROIs were applied.

At a deeper level, micro-tractography provides a glimpse of the microstructural organisation within an individual layer. For example, the three-dimensional description offered by tractography consistently reveals the presence of repeated intra-cortical connections between the granular layer and the molecular layer as short strands that merge almost orthogonally with longer strands representing the parallel fibres (Fig. 4). At this resolution, these reconstructions are likely to be representative of the anatomy of a small group of hundreds of granular cell axons that coherently run within each voxel. Importantly, these tractography reconstructions follow the anatomical trajectories of real fibres (i.e. granular cell axons) that as short strands bend almost orthogonally at the level of the T-junctions to continue as parallel fibres inside the molecular layer. Although Purkinje cell bodies may also play a role in this reconstruction, their large cytoplasm ( $\sim 50 \mu \mathrm{m}$ diameter) and lower density make them virtually undetectable inside the dominant diffusion component. In this figure, a first ROI was delineated around the white matter medullary layer to visualise only those streamlines passing through the white matter and the granular layer (green coloured). An exclusion ROI was used to exclude those streamlines reaching the molecular layer. Vice versa short strands and long strands (red coloured) were visualised selecting all streamlines passing through a ROI in the molecular layer. In this case, one exclusion ROI in the white matter medullary layer was used to exclude streamlines reaching the inner layer.

\section{Discussion}

Although a detailed cytoarchitectural description of the human brain by means of histology is long established, an integrated view of brain and cerebellar connections ranging from major white matter pathways to individual neurons or even small fascicles of a few hundred axons is still largely missing [22-24, 32]. In this preliminary study, we have shown that high-resolution MR diffusion imaging methods applied to fixed ex vivo human cerebellum have the potential to extend our ability to study brain connectivity, from a macroscopic description down to the mesoscale level, and at the same time provide results that can be directly compared with histological features observed at the microscale.

Conventional in vivo diffusion imaging applications acquire data with isotropic spatial resolutions of $2 \mathrm{~mm}(8 \mu \mathrm{l} / \mathrm{voxel})$. Data acquired in our experiment had a spatial resolution of $100 \times 100 \times 200 \mu \mathrm{m}(2 \mathrm{nl} /$ voxel $)$, equivalent to a voxel volume 


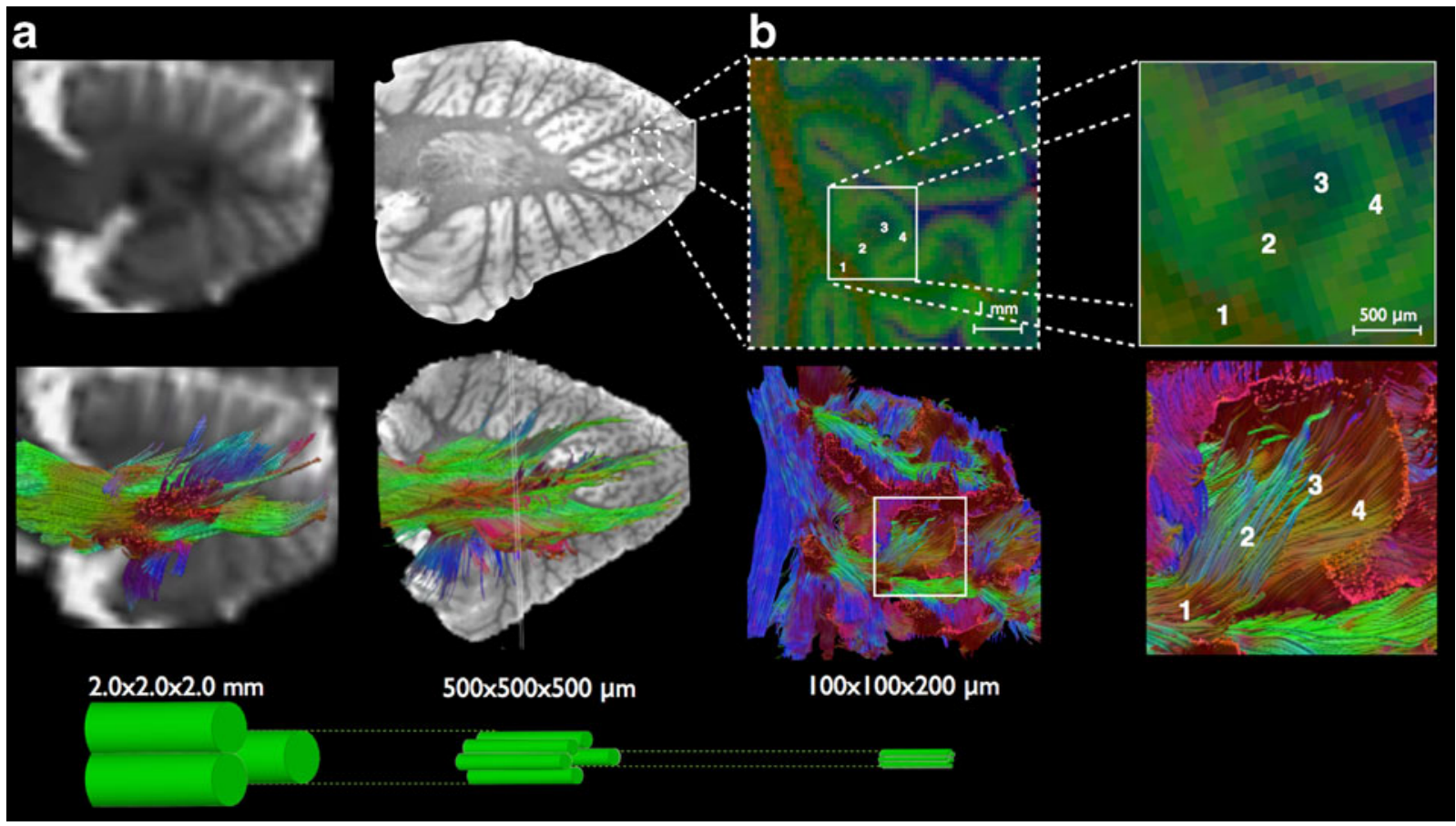

Fig. 3 Tractography from macro- to microscale. a A conventional clinical DTI scan at a resolution of $2 \times 2 \times 2 \mathrm{~mm}(8 \mu \mathrm{l} /$ voxel $)$ reveals the directionality of major fibre tracts overlaid onto the main macroscopic anatomical features of the cerebellum. On a fixed cerebellum acquired at $500 \times 500 \times 500 \mu \mathrm{m}(0.25 \mu \mathrm{l} /$ voxel, 32 times smaller voxel than clinical scan), it is possible to further focus on the cerebellum to reveal a far greater number of anatomical details and connections. $\mathbf{b}$
However, it is at a resolution of $100 \times 100 \times 200 \mu \mathrm{m}(2 \mathrm{nl} /$ voxel, 4,000 times smaller voxel than on a clinical scan) that it is possible to clearly observe mesoscale connectivity features of white matter and histological details of the cerebellum grey matter. The arbor vitae (1), intra-folia axonal projections (2), granular cell layer (3), as well as molecular cell layer (4) are all identifiable within each cerebellar folium using MR diffusion histology and micro-tractography reconstructions

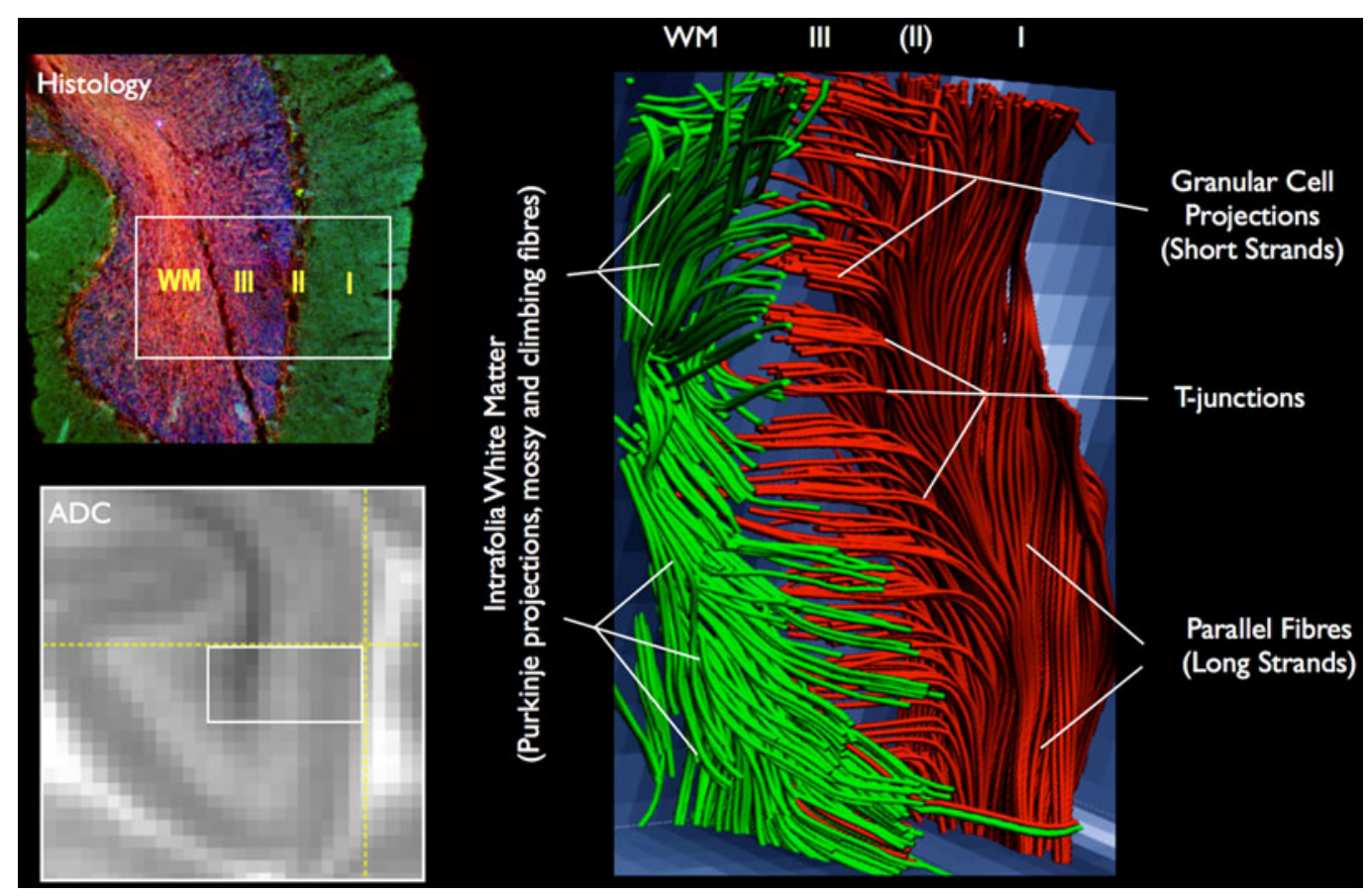

Fig. 4 Cytoarchitectural tractography. Features of the cerebellar intracortical connectivity are revealed by micro-tractography in a portion of cerebellar folium. White matter $(W M)$ trajectories propagate and disperse inside the granular layer $(I I I)$ of the folium. At this interface, short strands, reflecting probably the average orientation of granular cell axons, project to the molecular layer $(I)$ and merge with almost orthogonal and longer strands that consistently describe the dominant orientation of the parallel fibres $(I I)$ inside this layer 
4,000 times smaller than those acquired in clinical DTI scans. At this resolution, it was possible to start to investigate the cytoarchitectural basis of diffusion properties related to distinct histological components of cerebellar grey matter. Differences in ADC and FA between the white matter, granular layer and molecular layer were consistent with our knowledge of the microstructural and histological organisation of the cerebellum (for a review see [51]). Additionally, the comparison between MR diffusion histology maps and immunohistochemistry results showed a good agreement in identifying the same mesoscale features of the cerebellar cortex. We think that this approach, thanks to its ability to characterise cytoarchitectural organisation over relatively large brain samples, offers a new imaging tool for systematically investigating the microstructural organisation of different cortical layers and complex regions of the cerebellum and the entire human brain. By providing quantitative and layer-specific measurements of the microstructural tissue organisation, this approach can be directly applied to investigate pathologies and disorders that may involve selected group of cells or individual cortical layers. While offering new insights at a range of scales not currently accessible with conventional clinical MRI scanners, these results could potentially integrate and complement other modalities to allow better interpretation of in vivo clinical and functional results. Moreover, due to the non-destructive imaging procedure, this technique can allow histological analyses of the same imaged sample and the study of the direct correspondence between histopathological changes and macroscopic MRI changes in neurological and psychiatric disorders affecting the cerebellum and its connectivity (e.g. cerebellar ataxia, autism, and schizophrenia). This technique may potentially represent a new image-guided histological planning tool that could help neuropathologists to better locate and characterise pathological changes.

A unique feature of diffusion imaging is its ability to provide a three-dimensional description of the microstructural organisation of brain tissue and, over a larger scale, to identify how groups of axons integrate into larger fibre tracts that represent the intricate network of connections that link distant regions of the cerebellum [37, 44]. In this study, we were able to reconstruct the microstructural organisation of individual cerebellar folia and visualise how fibres from a major axonal bundle penetrate and disperse inside the granular cell layer. From here, we were also able to reconstruct details of the intra-cortical organisation of the cerebellum, showing streamlines describing the average trajectories of a few hundred granular cell axons that orthogonally merge with components representing parallel fibres running inside the molecular layer. To our knowledge, this represents the first attempt of intra-cortical tractography in the human brain using MR diffusion imaging techniques.

Compared to low spatial resolution datasets from clinical DTI acquisitions, results of this study were able to resolve distinct anatomical features and details of connectivity that are normally mixed within individual voxels [32]. However, it is essential to consider that, even at the mesoscale, diffusion-based tractography reconstructions only provide an indirect representation of the underlying biological organisation and not the direct visualisations of biological connections, such as real axons or fascicles. For example, at the spatial resolution employed in this study, the diffusion signal of a single voxel in the granular cell layer is still an average measurement derived from a large number of individual axons and cell bodies that cannot be quantified individually with our current approach. Partial volume and inadequate diffusion modelling may still produce artefacts or incomplete reconstructions [52]. The ability to visualize only one of the branch of the bifurcating parallel fibres at the level of the T-junctions, is an example of the inadequacy of current tractography algorithms. A further increase in spatial resolution [46], new tractography algrorithms and new diffusion imaging approaches, including multi-compartmental modelling [53-55] or multi-fibre orientation estimators such as High Angular Resolution Diffusion Imaging and Diffusion Spectrum Imaging techniques [16, 56-58], may be required to resolve multiple crossing fibres and the remaining partial volume characteristics of cytoarchitecturally mixed voxels.

Another important limitation is the long acquisition time of the formalin-fixed cerebellum that required an uninterrupted scan time of approximately 6 days. This acquisition clearly represents a special case that cannot represent the standard acquisition protocols applicable to a large number of samples. Previous diffusion imaging studies on animals and also human samples provide important guidelines on how to sensibly reduce scan times and improve signal quality [59-62]. Our group is currently investigating these approaches, and future work will optimise novel and more specific sample preparation procedures together with new acquisition strategies.

The ability to visualise the three-dimensional organisation of the mesoscale represents a unique feature of diffusion imaging and offers a new approach for investigating the anatomical basis of connectivity within the cerebellum and its complex functions [16]. The role of cortico-cerebellar connectivity in cognition, for instance, is still under investigation and represents a topic of intense debate and interest. Data on the precise anatomy of the intracerebellar connectivity in humans and the pathways that relay information from the cerebellum to associative cortex could help to confirm the involvement of the cerebellum in higher cognitive functions [31, 63, 64]. Mapping white matter pathways from the macro- to microscale could help create a more detailed atlas of the cytoarchitectural organisation and connectivity not only of the human cerebellum [65-67] but also of the cerebral cortex in general [68]. 


\section{Conclusions}

A detailed analysis of cerebellar connectivity could reveal unique features of human anatomy and address important questions related to the role of the cerebellum in higher cognitive functions. MR diffusion histology and microtractography represent two novel methods for obtaining a three-dimensional description of tissue cytoarchitecture at the mesoscale level. These methods produce quantitative information that, coupled with high-resolution visualisation of small fibres, can bridge the gap between large-scale network mapping and microscopic histology. We believe that this approach, applied to fixed post-mortem samples, represents an essential step forward in the understanding of the human cerebellum and its connectivity with brainstem nuclei and cerebral cortex. Future applications of these methods promise to have practical implications in the diagnosis and treatment of cerebellar disorders.

Acknowledgments The authors would like to thank Prof. Steve C.R. Williams for his continued support and stimulation to push the boundaries of imaging science, as well as Dr. Po-Wah So, the manager of the preclinical imaging unit at KCL, and the British Heart Foundation for funding the 7T MRI scanner. MM gratefully acknowledges funding support by NIBIB (1 P20 EB007076-01), the UK Medical Research Council (G0802552 \& G0800846) and the 7th framework from the European Union (201842-ENCITE). FDA, IB, MC acknowledge support from the NIHR Biomedical Research Centre for Mental Health at the South London and Maudsley NHS Foundation Trust.

Conflict of Interest The authors declare no conflict of interest.

Open Access This article is distributed under the terms of the Creative Commons Attribution License which permits any use, distribution, and reproduction in any medium, provided the original author(s) and the source are credited.

\section{References}

1. Golgi C. Sulla struttura della sostanza grigia del cervello. Gazzetta Med Ital Lombardia. 1873;6:244-6.

2. Pannese E. The Golgi stain: invention, diffusion and impact on neurosciences. J Hist Neurosci. 1999;8(2):132-40.

3. Ramon y Cajal S. The Croonian Lecture. La fine structure des centres nerveux. Proc R Soc Lond. 1894;55:444-67.

4. Glickstein M, Strata P, Voogd J. Cerebellum: history. Neuroscience. 2009;162(3):549-59.

5. Dow RS, Moruzzi G. The physiology and pathology of the cerebellum. Minneapolis: University of Minnesota Press; 1958.

6. Marr D. A theory of the cerebellar cortex. J Physiol. 1969;202:43770 .

7. Albus JS. A theory of cerebellar function. Math Biosci. 1971;10:2561.

8. Manto M, Bower JM, Conforto AB, Delgado-García JM, da Guarda SN F, Gerwig M, et al. Consensus paper: roles of the cerebellum in motor control-the diversity of ideas on cerebellar involvement in movement. Cerebellum. 2012;11(3):35265 .
9. Strata P. David Marr's theory of cerebellar learning: 40 years later. J Physiol. 2009;587(23):5519-20.

10. Dum RP, Strick PL. An unfolded map of the cerebellar dentate nucleus and its projections to the cerebral cortex. J Neurophysiol. 2003;89:634-9.

11. Kelly RM, Strick PL. Cerebellar loops with motor cortex and prefrontal cortex of a nonhuman primate. J Neurosci. 2003;23:8432-44.

12. Schmahmann J, Sherman JC. The cerebellar cognitive affective syndrome. Brain. 1998;121:561-79.

13. Schmahmann JD, Weilburg JB, Sherman JC. The neuropsychiatry of the cerebellum-insights from the clinic. Cerebellum. 2007;6:254-67.

14. Deoni SCL, Catani M. Visualization of the deep cerebellar nuclei using quantitative $\mathrm{T} 1$ and rho magnetic resonance imaging at 3 Tesla. NeuroImage. 2007;37(4):1260-6.

15. Marques JP, van der Zwaag W, Granziera C, Krueger G, Gruetter R. Cerebellar cortical layers: in vivo visualization with structural highfield-strength MR imaging. Radiology. 2010;254(3):942-8.

16. Granziera C, Schmahmann JD, Hadjikhani N, Meyer H, Meuli R, Wedeen VJ, et al. Diffusion spectrum imaging shows the structural basis of functional cerebellar circuits in the human cerebellum in vivo. PLoS One. 2009;4:e5101.

17. Catani M, Jones DK, Daly E, Embiricos N, Deeley Q, Pugliese L, et al. Altered cerebellar feedback projections in Asperger syndrome. NeuroImage. 2008;41(4):1184-91.

18. Lee S-K, Kim DI, Kim J, Kim DJ, Kim HD, Kim DS, et al. Diffusion-tensor MR imaging and fiber tractography: a new method of describing aberrant fiber connections in developmental CNS anomalies. Radiographics. 2005;25:53-65.

19. Okugawa G, Nobuhara K, Minami T, Takase K, Sugimoto T, Saito $\mathrm{Y}$, et al. Neural disorganization in the superior cerebellar peduncle and cognitive abnormality in patients with schizophrenia: a diffusion tensor imaging study. Prog Neuro-Psychopharmacol Biol Psychiatry. 2006;30:1408-12.

20. Taoka T, Kin T, Nakagawa H, Hirano M, Sakamoto M, Wada T, et al. Diffusivity and diffusion anisotropy of cerebellar peduncles in cases of spinocerebellar degenerative disease. NeuroImage. 2007;37:387-93.

21. Fatemi SH, Aldinger KA, Ashwood P, Bauman ML, Blaha CD, Blatt GJ, et al. Consensus paper: pathological role of the cerebellum in autism. Cerebellum. 2012;11(3):777-807.

22. DeFelipe J. From the connectome to the synaptome: an epic love story. Science. 2010;330:1198-201.

23. Lichtman JW, Denk W. The big and the small: challenges of imaging the brain's circuits. Science. 2011;334:618-23.

24. Sporns O, Tononi G, Kotter R. The human connectome: a structural description of the human brain. PLoS Comput Biol. 2005; $1: \mathrm{e} 42$.

25. Kobbert C, Apps R, Bechmann I, Lanciego JL, Mey J, Thanos S. Current concepts in neuroanatomical tracing. Prog Neurobiol. 2000;62:327-51.

26. Boldogkoi Z, Sik A, Denes A, Reichart A, Toldi J, Gerendai I, et al. Novel tracing paradigms - genetically engineered herpesviruses as tools for mapping functional circuits within the CNS: present status and future prospects. Prog Neurobiol. 2004;72:417-45.

27. Wouterlood FG, Vinkenoog M, van den Oever M. Tracing tools to resolve neural circuits. Network. 2002;13:327-42.

28. Pautler RG, Koretsky AP. Tracing odor-induced activation in the olfactory bulbs of mice using manganese-enhanced magnetic resonance imaging. NeuroImage. 2002;16:441-8.

29. Racette BA, Aschner M, Guilarte TR, Dydak U, Criswell SR, Zheng W. Pathophysiology of manganese-associated neurotoxicity. Neurotoxicology. 2012;33(4):881-6.

30. Balsters JH, Cussans E, Diedrichsen J, Phillips KA, Preuss TM, Rilling JK, et al. Evolution of the cerebellar cortex: the selective expansion of prefrontal-projecting cerebellar lobules. NeuroImage. 2010;49:2045-52. 
31. Rathelot JA, Strick PL. Subdivisions of primary motor cortex based on cortico-motoneuronal cells. Proc Natl Acad Sci U S A. 2009;106:918-23.

32. Mesulam M. The evolving landscape of human cortical connectivity: facts and inferences. NeuroImage. 2012;62(4):2182-9.

33. Swanson LW. Mapping the human brain: past, present, and future. Trends Neurosci. 1995;18:471-4.

34. Stoodley CJ, Schmahmann JD. Functional topography in the human cerebellum: a meta-analysis of neuroimaging studies. NeuroImage. 2009;44:489-501.

35. Peterburs J, Bellebaum C, Koch B, Schwarz M, Daum I. Working memory and verbal fluency deficits following cerebellar lesions: relation to interindividual differences in patient variables. Cerebellum. 2010;9(3):375-83.

36. Stoodley CJ. The cerebellum and cognition: evidence from functional imaging studies. Cerebellum. 2012;11(2):352-65.

37. Jones DK. Studying connections in the living human brain with diffusion MRI. Cortex. 2008;44:936-52.

38. Basser PJ, Mattiello J, Le Bihan D. MR diffusion tensor spectroscopy and imaging. Biophys J. 1994;66:259-67.

39. Mori S, van Zijl PCM. Fiber tracking: principles and strategies-a technical review. NMR Biomed. 2002;15:468-80.

40. Basser PJ, Pajevic S, Pierpaoli C, Duda J, Aldroubi A. In vivo fiber tractography using DT-MRI data. Magn Reson Med. 2000;44:625-32.

41. Catani M, Howard RJ, Pajevic S, Jones DK. Virtual in vivo interactive dissection of white matter fasciculi in the human brain. NeuroImage. 2002;17(1):77-94.

42. Thiebaut de Schotten M, Dell'Acqua F, Forkel S, Simmons A, Vergani F, Murphy D, et al. A lateralized brain network for visuospatial attention. Nat Neurosci. 2011;14(10):1245-6.

43. Wiegell MR, Larsson HBW, Wedeen VJ. Fiber crossing in human brain depicted with diffusion tensor MR imaging. Radiology. 2000;217:897-903.

44. Seehaus AK, Roebroeck A, Chiry O, Kim DS, Ronen I, Bratzke H, et al. Histological validation of DW-MRI tractography in human postmortem tissue. Cereb Cortex. 2013;23(2):442-50.

45. Leergaard TB, White NS, de Crespigny AJ, Bolstad I, D'Arceuil HE, Bjaalie JG, et al. Quantitative histological validation of diffusion MRI fiber orientation distributions in the rat brain. PLoS One. 2010;e8595(1).

46. Flint JJ, Hansen B, Fey M, Schmidig D, King MA, VestergaardPoulsen $\mathrm{P}$, et al. Cellular-level diffusion tensor microscopy and fiber tracking in mammalian nervous tissue with direct histological correlation. NeuroImage. 2010;52:556-61.

47. Mcnab JA, Jbabdi S, Deoni SCL, Douaud G, Behrens TEJ, Miller KL. High resolution diffusion-weighted imaging in fixed human brain using diffusion-weighted steady state free precession. NeuroImage. 2009;46(3):775-85.

48. Miller KL, Stagg CJ, Douaud G, Jbabdi S, Smith SM, Behrens TEJ, et al. Diffusion imaging of whole, post-mortem human brains on a clinical MRI scanner. NeuroImage. 2011;57(1):167-81.

49. Shepherd TM, Ozarslan E, Yachnis AT, King MA, Blackband SJ. Diffusion tensor microscopy indicates the cytoarchitectural basis for diffusion anisotropy in the human hippocampus. Am J Neuroradiol. 2007;28:958-64.

50. Jones DK, Basser PJ. Squashing peanuts and smashing pumpkins: how noise distorts diffusion-weighted MR data. Magn Reson Med. 2004;52(5):979-93.
51. Apps R, Garwicz M. Anatomical and physiological foundations of cerebellar information processing. Nat Rev Neurosci. 2005;6:297311.

52. Dell'Acqua F, Catani M. Structural human brain networks: hot topics in diffusion tractography. Curr Opin Neurol. 2011;25(4):375-83.

53. Assaf Y, Basser PJ. Composite hindered and restricted model of diffusion (CHARMED) MR imaging of the human brain. NeuroImage. 2005;27:48-58.

54. Alexander DC. A general framework for experiment design in diffusion MRI and its application in measuring direct tissuemicrostructure features. Magn Reson Med. 2008;60(2):439-48.

55. Jespersen SN, Kroenke CD, Ostergaard L, Ackerman JJ, Yablonskiy DA. Modeling dendrite density from magnetic resonance diffusion measurements. NeuroImage. 2007;34:1473-86.

56. Wedeen VJ, Hagmann P, Tseng WYI, Reese TG, Weisskoff RM. Mapping complex tissue architecture with diffusion spectrum magnetic resonance imaging. Magn Reson Med. 2005;54(6):1377-86.

57. Tournier JD, Calamante F, Gadian DG, Connelly A. Direct estimation of the fiber orientation density function from diffusionweighted MRI using spherical deconvolution. NeuroImage. 2004;23:1176-85.

58. Dell'Acqua F, Scifo P, Rizzo G, Catani M, Simmons A, Scotti G, et al. A modified damped Richardson-Lucy algorithm to reduce isotropic background effects in spherical deconvolution. NeuroImage. 2010;49(2):1446-58.

59. Shepherd TM, Flint JJ, Thelwall PE, Stanisz GJ, Mareci TH, Yachnis AT, et al. Postmortem interval alters the water relaxation and diffusion properties of rat nervous tissue - implications for MRI studies of human autopsy samples. NeuroImage. 2009;44:820-6.

60. D'Arceuil HE, Crespigny AJ. The effects of brain tissue decomposition on diffusion tensor imaging and tractography. NeuroImage. 2007;36:64-8.

61. D'Arceuil HE, Westmoreland S, de Crespigny AJ. An approach to high resolution diffusion tensor imaging in fixed primate brain. NeuroImage. 2007;35:553-65.

62. Dyrby TB, Baare WFC, Alexander DC, Jelsing J, Garde E, Søgaard LV. An ex vivo imaging pipeline for producing high-quality and high-resolution diffusion-weighted imaging datasets. Hum Brain Mapp. 2011;32:544-63.

63. Ramnani N. The primate cortico-cerebellar system: anatomy and function. Nat Rev Neurosci. 2006;7:511-22.

64. Sultan F, Augath M, Hamodeh S, Murayama Y, Oeltermann A, Rauch A, et al. Unravelling cerebellar pathways with high temporal precision targeting motor and extensive sensory and parietal networks. Nature Commun. 2012;3:924

65. Diedrichsen J, Balsters JH, Flavell J, Cussans E, Ramnani N. A probabilistic MR atlas of the human cerebellum. NeuroImage. 2009;46:39-46.

66. Saksena S, Husain N, Das V, Pradhan M, Trivedi R, Srivastava $\mathrm{S}$, et al. Diffusion tensor imaging in the developing human cerebellum with histologic correlation. Int J Dev Neurosci. 2008;26:705-11.

67. Annese J. The importance of combining MRI and large-scale digital histology in neuroimaging studies of brain connectivity and disease. Front Neuroinformatics. 2012;6:13.

68. Catani M, Thiebaut de Schotten M, Slater D, Dell'Acqua F. Connectomic approaches before the connectome. Neuroimage. 2013. doi:10.1016/j.neuroimage.2013.05.109. 